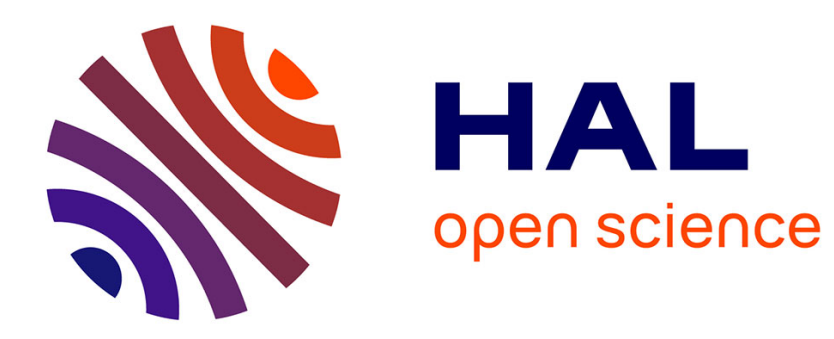

\title{
Derivation of a viscous KP equation including surface tension, and related equations
}

Hervé Le Meur

\section{To cite this version:}

Hervé Le Meur. Derivation of a viscous KP equation including surface tension, and related equations. IMA Journal of Applied Mathematics, 2018, 83 (3). hal-01146171v2

\section{HAL Id: hal-01146171 \\ https://hal.science/hal-01146171v2}

Submitted on 5 Nov 2015

HAL is a multi-disciplinary open access archive for the deposit and dissemination of scientific research documents, whether they are published or not. The documents may come from teaching and research institutions in France or abroad, or from public or private research centers.
L'archive ouverte pluridisciplinaire HAL, est destinée au dépôt et à la diffusion de documents scientifiques de niveau recherche, publiés ou non, émanant des établissements d'enseignement et de recherche français ou étrangers, des laboratoires publics ou privés. 


\title{
Derivation of a viscous KP equation including surface tension, and related equations
}

\author{
Hervé V.J. Le Meur ${ }^{1 *}$ \\ ${ }^{1}$ Laboratoire de Mathématiques d'Orsay, Univ. Paris-Sud, \\ CNRS, Université Paris-Saclay, 91405 Orsay, France. \\ Herve. LeMeur@math. u-psud.fr
}

October 2015

\begin{abstract}
The aim of this article is to derive surface wave models in the presence of surface tension and viscosity. Using the Navier-Stokes equations with a free surface, flat bottom and surface tension, we derive the viscous 2D Boussinesq system with a weak transverse variation. The assumed transverse variation is on a larger scale than along the main propagation direction. This Boussinesq system is proved to be consistent with the Navier-Stokes equations. This system is only an intermediate result that enables us to derive the Kadomtsev-Petviashvili (KP) equation which is a 2D generalization of the KdV equation. In addition, we get the 1D KdV equation, and lastly the Boussinesq equation. All these equations are derived for non-vanishing initial conditions.
\end{abstract}

Subject Class: 74J15, 35Q30, 76M45, 35Q35

Keywords: water waves, shallow water, Boussinesq system, viscosity, KdV equation, surface tension, KP

\section{Introduction}

\subsection{Motivation}

Understanding the evolution of water waves is a longstanding problem. For instance, Stokes already discussed a precise wave at least 130 years ago [18] and his name has been given to this wave. Such a wave was still discussed more than one century later [3] !

One of the striking phenomena is that there exist surface waves that travel almost without modification. Such a behavior is an impetus to investigate the dynamics of these waves.

\footnotetext{
*The author wants to thank Luc Molinet and Jean-Claude Saut for fruitful discussions.
} 
The first motivation is to understand how they may either disperse or dissipate and yet not vanish. It was discovered that there is a regime in which nonlinearity compensates dispersion (with no dissipation).

Another motivation is more technical. Should a wave keep its shape for a long time without vanishing, it could be used in telecommunication without requiring repeaters. Such a new technology could drastically reduce the cost of telecommunications.

Beyond mathematical and industrial considerations, mathematicians and physicists model the water waves with a fluid flow in a channel of (often) flat bottom, without meniscus (appart in [14]). Since the Euler or Navier-Stokes equations are rather prone to faithfully model such a fluid, one could be satisfied with either of these models. Yet, the Direct Numerical Simulation is too expensive and asymptotic models are required. In such models, one assumes a regime of dimensionless parameters' smallness and makes expansions of the equations to derive a simplified model with less fields and less dimensions.

\section{$1.2 \quad$ Literature}

The techniques are very different depending on whether one chooses Euler or NavierStokes as a model for the fluid.

On the one hand, for an inviscid flow (Euler), one assumes very often irrotationality. Then one may use a potential function and a Dirichlet to Neumann operator in the Zakharov-Craig-Sulem formulation. Numerous articles use this formulation and a review of the mathematical proofs has been published [11]. Some authors use a velocity-pressure formulation, but they still assume the irrotationnality assumption [4]. The derivation of Kadomtsev-Petviashvili (KP) assumes a predominant propagation direction along $x$ and a weakly transverse propagation along $y$. It is initially done for an inviscid fluid in [6] and the justification of this approximation is done in [11] (subsection 7.2). In [10], D. Lannes proves that the sum of one wave propagating to the right and one to the left, both obeying a KP equation converges (when $\varepsilon$ tends to zero) on $\left[0, T_{0} / \varepsilon\right]$ to a function consistent with the Boussinesq system, meaning that it is a solution, should it be only up to $O\left(\varepsilon^{2}\right)$.

Various difficulties must be considered with these surface wave models. Firstly two scales of the transverse velocity are choosen in the literature. Either one assumes the transverse velocity to be $O(1)$ or $O(\sqrt{\varepsilon})$. This problem was raised in Remark 3 of [9] and here in Remark 3. It leads to different Boussinesq systems.

In [15], the authors prove that a dimensionless water wave system in an infinite strip under the influence of gravity and surface tension has a unique solution on $[0, T / \varepsilon]$. More precisely, if the initial solution is sufficiently regular and small $(O(\sqrt{\varepsilon}))$, there exists a solution, on this time interval $[0, T / \varepsilon]$, that will remain small $(O(\sqrt{\varepsilon}))$. In [16], the same authors prove that on the same time interval, these solutions can be accurately approximated by sums of solutions of two decoupled KP equations. It enhances the results of [9] by taking surface tension and a variable bottom into consideration.

The Boussinesq equation is a generalization of the KdV equation for waves moving both to the right and to the left. From the Boussinesq system, instead of changing to a frame moving to the right as is done for $\mathrm{KdV}$, we remain in the same coordinate system and get higher order equation (perturbed wave equation), as is done in [5] (p. 216-219). 
On the other hand, when one assumes the fluid to be viscous, one may not use the potential function. Moreover the number of boundary conditions changes then. Viscosity has been considered in water waves since 1895 by Boussinesq [1]. The dynamic of viscous water waves on finite depth was investigated more recently by Kakutani and Matsuuchi [7]. They derived the viscous KdV equation from vanishing initial conditions but made the error of using a Fourier transform in time while the problem is of Cauchy type.

Liu and Orfila [13], and the coauthors of P.L.-F. Liu in subsequent articles, investigated the viscous Boussinessq system with vanishing initial condition, and validated their $\mathrm{KdV}$ equation by some experiments. For instance they experimentally proved the reverse flow in the boundary layer, which is predicted by theory.

Although it was not done in Sobolev spaces, the derivation of the viscous Boussinesq system (1D and isotropic 2D) and 1D KdV equation with a non-vanishing initial condition was done in [12] without surface tension. It was partially the goal of [12] not to rely on the irrotationnality assumption so as to derive the Boussinesq system and KdV equation for a viscous fluid. This could be achieved thanks to the fact that one of the equations contains $u_{z}=O(\varepsilon)$ which is the zeroth order of the irrotationnality assumption. In [12], a more detailed bibliography was given and the reason why all the preceding articles did not derive the same KdV equation is explained.

In this article, we intend to cross the results for a viscous fluid with some results concerning inviscid fluids (KP, Boussinesq equation, surface tension).

The outline of this article is as follows. In section 2, we present the equations and state the viscous Boussinesq system in $2 \mathrm{D}$ with surface tension and a predominant propagation direction. This result is needed to get the new results on viscous $1 \mathrm{D} \mathrm{KdV}$ equations in section 3, the viscous $\mathrm{KP}$ equation (2D generalization of $\mathrm{KdV}$ with a weak transverse propagation) derived in section 4 , and the viscous Boussinesq equation derived in section 5. Finally, we justify that our Boussinesq system is consistent with the Navier-Stokes equations in Section 6. All these derivations are done for a viscous fluid with surface tension and non-vanishing initial conditions.

\section{2D geometry with viscosity and surface tension}

Below, we define the geometry, write the equations, and then make these equations dimensionless (subsection 2.1). These equations model a propagation predominantly in the $x$-direction. This will enable us to make a linear analysis with the dispersion relation and an asymptotic of the phase velocity in subsection 2.2. A discussion of the relevance of viscosity and surface tension will be given in subsection 2.3. We will then use the results of [12], where the 1D viscous isotropic Boussinesq system and KdV equation are derived, so as to state the weakly transverse $2 \mathrm{D}$ viscous Boussinesq system (subsection 2.4). Our present derivation includes surface tension, viscosity and a propagation mainly along $x$ and weakly along $y$. Since the already published results are very close, most proofs are omitted. 


\subsection{Navier-Stokes equations}

Let $\tilde{\mathbf{u}}=(\tilde{u}, \tilde{v}, \tilde{w})$ be the velocity of a fluid in a 3 -D domain $\tilde{\Omega}=\left\{(\tilde{x}, \tilde{y}, \tilde{z}) /(\tilde{x}, \tilde{y}) \in \mathbb{R}^{2}, \tilde{z} \in\right.$ $(-h, \tilde{\eta}(\tilde{x}, \tilde{y}, \tilde{t}))\}$. So we assume the bottom is flat and the free surface is characterized by $\tilde{z}=\tilde{\eta}(\tilde{x}, \tilde{y}, \tilde{t})$ with $\tilde{\eta}(\tilde{x}, \tilde{y}, \tilde{t})>-h$ (the bottom does not get dry). Let $\tilde{p}$ be the pressure and $\tilde{\mathbf{D}}[\tilde{\mathbf{u}}]$ the symmetric part of the velocity gradient. The dimensionless domain is illustrated in Fig. 1. We also denote $\rho$ the density of the fluid, $\nu$ the viscosity of the fluid, $g$ the

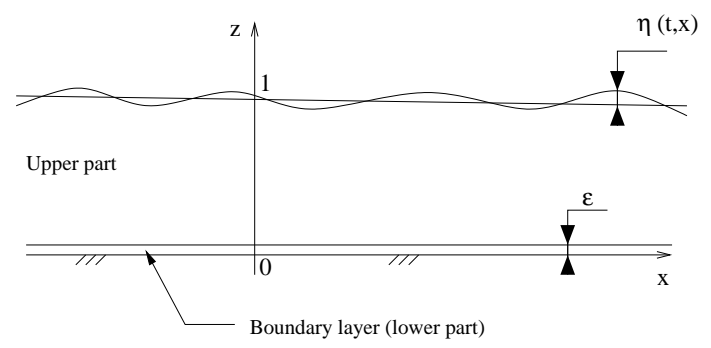

Figure 1: The dimensionless domain

gravitational acceleration, $\mathbf{k}$ the unit vertical vector, $\mathbf{n}$ the outward unit normal to the upper frontier of $\tilde{\Omega}, \sigma$ the surface tension coefficient, and $\tilde{p}_{a t m}$ the atmospheric pressure. The original system reads:

$$
\begin{cases}\rho\left(\frac{\partial \tilde{\mathbf{u}}}{\partial \tilde{t}}+\tilde{\mathbf{u}} . \tilde{\nabla} \tilde{\mathbf{u}}\right)-\nu \tilde{\Delta} \tilde{\mathbf{u}}+\tilde{\nabla} \tilde{p}=-\rho g \mathbf{k} & \text { in } \tilde{\Omega}, \\ \widetilde{\operatorname{div} \tilde{\mathbf{u}}=0} & \text { in } \tilde{\Omega}, \\ (-\tilde{p} \mathbf{I}+2 \nu \tilde{\mathbf{D}}[\tilde{\mathbf{u}}]) \cdot \mathbf{n}=-\tilde{p}_{a t m} \mathbf{n}+\frac{\sigma}{R} \mathbf{n} & \text { on } \tilde{z}=\tilde{\eta}(\tilde{x}, \tilde{t}), \\ \tilde{\eta}_{\tilde{t}}+\tilde{u} \tilde{\eta}_{\tilde{x}}+\tilde{v} \tilde{\eta}_{\tilde{y}}-\tilde{w}=0 & \text { on } \tilde{z}=\tilde{\eta}(\tilde{x}, \tilde{t}), \\ \tilde{\mathbf{u}}=0 & \text { on } \tilde{z}=-h,\end{cases}
$$

where we write the second order tensors and the vectors with bold letters. The differentiated functions are denoted either $\partial f / \partial x, f_{x}$, or $\partial_{x} f$. The surface tension term includes $R$ given by geometrical computations:

$$
\frac{1}{R}=\frac{\left(1+\tilde{\eta}_{\tilde{y}}^{2}\right) \tilde{\eta}_{\tilde{x} \tilde{x}}+\left(1+\tilde{\eta}_{\tilde{x}}^{2}\right) \tilde{\eta}_{\tilde{y} \tilde{y}}-2 \tilde{\eta}_{\tilde{x}} \tilde{\eta}_{\tilde{y}} \tilde{\eta}_{\tilde{x} \tilde{y}}}{\left(1+\tilde{\eta}_{\tilde{x}}^{2}+\tilde{\eta}_{\tilde{y}}^{2}\right)^{3 / 2}}
$$

Of course, we need to add an initial condition and conditions at infinity.

So as to get dimensionless fields and variables, we need to choose a characteristic horizontal length $l$ which is the wavelength along the propagation direction, a characteristic vertical length $h$ which is the water's height, and the amplitude $A$ of the propagating perturbation. Moreover, let $U, V, W, P$ be the characteristic horizontal velocity along $x$, horizontal velocity along $y$, vertical velocity and pressure respectively. Since the zeroth order equation is a wave equation of dispersion relation $\omega=c_{0} k_{x}\left(1+k_{y}^{2} / 2 / k_{x}^{2}\right)+o\left(k_{y}^{2}\right)$, one may infer that a good scaling for the $y$ direction is if the extra term is of the same order of magnitude as $\varepsilon$. So $k_{y}=O\left(\varepsilon^{1 / 2}\right)$ and the interesting scale for $\tilde{y}$ is $O\left(\varepsilon^{-1 / 2}\right)$ more 
than for the $x$ direction. We may then define:

$$
\begin{aligned}
& c_{0}=\sqrt{g h}, \varepsilon=\frac{A}{h}, \beta=\frac{h^{2}}{l^{2}}, U=\varepsilon c_{0}, V=\varepsilon c_{0}, \\
& W=\sqrt{\varepsilon} c_{0}, P=\rho g A, \operatorname{Re}=\frac{\rho c_{0} h}{\nu}, \mathrm{Bo}=\frac{\rho g l^{2}}{\sigma}
\end{aligned}
$$

where $c_{0}$ is the phase velocity. Then, one may make the fields dimensionless and unscaled:

$$
\tilde{u}=U u, \tilde{v}=V v, \tilde{w}=W w, \tilde{p}=\tilde{p}_{a t m}-\rho g \tilde{z}+P p, \tilde{\eta}=A \eta,
$$

and the variables too:

$$
\tilde{x}=l x, \tilde{y}=\varepsilon^{-1 / 2} l y, \tilde{z}=h(z-1), \tilde{t}=t l / c_{0} .
$$

We also make the Boussinesq approximation ( $\beta$ and $\varepsilon$ of the same order of magnitude) and even $\beta=\varepsilon$. Notice that we take the scale used by [9] $\left(V=\varepsilon c_{0}\right)$ and not the one taken by [5] $\left(V=\varepsilon^{3 / 2} c_{0}\right)$. The difference will be highlighted in Remark 3 .

With these definitions, the new system with the new fields, variables and outward unit normal, still denoted $\mathbf{n}$, writes in the new domain $\Omega_{t}=\left\{(x, y, z),(x, y) \in \mathbb{R}^{2}, z \in\right.$ $(0,1+\varepsilon \eta(x, y, t))\}$ (see Figure 1$)$ :

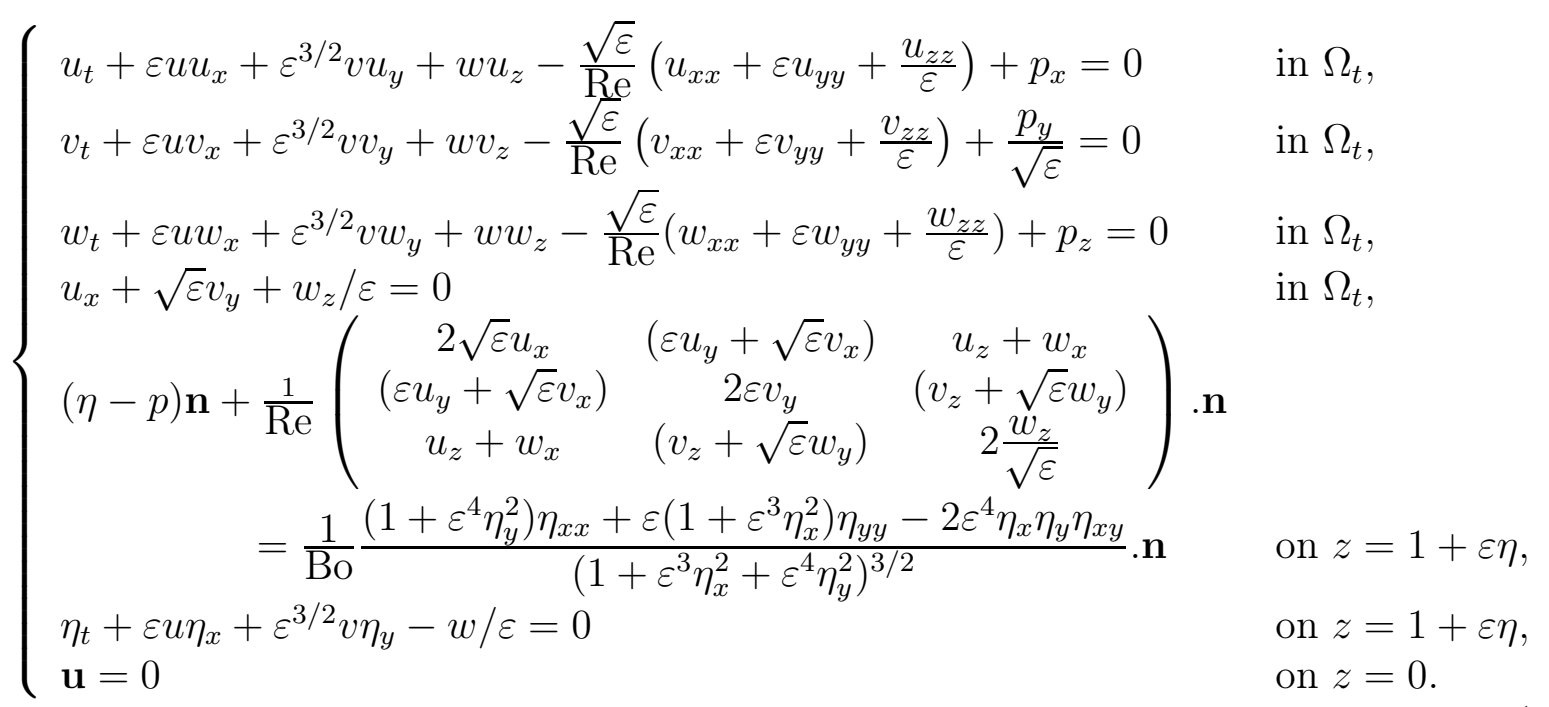

Simple computations give the outward non-unit normal $\mathbf{n}=\left(-\varepsilon \eta_{x},-\varepsilon \eta_{y}, 1\right)$.

\subsection{Linear theory}

The whole subsection below is a straightforward modification of the case of a viscous fluid without surface tension, fully studied in [12]. 


\subsubsection{Dispersion relation}

We are looking for small fields. So we linearize the system (3). The dynamic condition at the free boundary can then be stated more explicitely. We get:

$$
\begin{cases}u_{t}-\frac{\sqrt{\varepsilon}}{\operatorname{Re}}\left(u_{x x}+\varepsilon u_{y y}+u_{z z} / \varepsilon\right)+p_{x}=0 & \text { in } \mathbb{R}^{2} \times[0,1], \\ v_{t}-\frac{\sqrt{\varepsilon}}{\operatorname{Re}}\left(v_{x x}+\varepsilon v_{y y}+v_{z z} / \varepsilon\right)+\sqrt{\varepsilon} p_{y}=0 & \text { in } \mathbb{R}^{2} \times[0,1], \\ w_{t}-\frac{\sqrt{\varepsilon}}{\operatorname{Re}}\left(w_{x x}+\varepsilon w_{y y}+w_{z z} / \varepsilon\right)+p_{z}=0 & \text { in } \mathbb{R}^{2} \times[0,1], \\ u_{x}+\sqrt{\varepsilon} v_{y}+w_{z} / \varepsilon=0 & \text { in } \mathbb{R}^{2} \times[0,1], \\ \left(u_{z}+w_{x}\right) / \operatorname{Re}=0 & \text { on } z=1, \\ \left(v_{z}+\sqrt{\varepsilon} w_{y}\right) / \operatorname{Re}=0 & \text { on } z=1, \\ (\eta-p)+\frac{2 w_{z}}{\operatorname{Re} \sqrt{\varepsilon}}=\frac{1}{\mathrm{Bo}}\left(\eta_{x x}+\varepsilon \eta_{y y}\right) & \text { on } z=1, \\ \eta_{t}-w / \varepsilon=0 & \text { on } z=1, \\ \mathbf{u}=0 & \text { on } z=0 .\end{cases}
$$

In order to eliminate the pressure, we differentiate $(4)_{1}$ with respect to $z$ and $(4)_{3}$ with respect to $x$ and compute their difference so as to simplify $p_{x z}$. Symmetrically, we differentiate $(4)_{2}$ with respect to $z$ and $(4)_{3}$ with respect to $y$ and compute their difference (with a $\sqrt{\varepsilon}$ coefficient). Pressure is eliminated in two equations on three:

$$
\begin{aligned}
& u_{z t}-w_{x t}-\frac{\sqrt{\varepsilon}}{\operatorname{Re}}\left(\left(u_{x x z}-w_{x x x}\right)+\varepsilon\left(u_{y y z}-w_{y y x}\right)+\left(u_{z z z}-w_{x z z}\right) / \varepsilon\right)=0, \\
& v_{z t}-\sqrt{\varepsilon} w_{y t}-\frac{\sqrt{\varepsilon}}{\operatorname{Re}}\left(\left(v_{x x z}-\sqrt{\varepsilon} w_{x x y}\right)+\varepsilon\left(v_{y y z}-\sqrt{\varepsilon} w_{y y y}\right)+\left(v_{z z z}-\sqrt{\varepsilon} w_{y z z}\right) / \varepsilon\right)=0 .
\end{aligned}
$$

So as to eliminate $u$ and $v$ thanks to the incompressibility $(4)_{4}$ from the previous system, we differentiate the first equation with respect to $x$ and the second with respect to $y$. After some simplifications, a good combination of the two gives (thanks to $\left.(4)_{4}\right)$ :

$$
\left(\partial_{z}^{2}+\varepsilon \partial_{x}^{2}+\varepsilon^{2} \partial_{y}^{2}\right)\left(-\operatorname{Re} \sqrt{\varepsilon} \partial_{t}+\left(\partial_{z}^{2}+\varepsilon \partial_{x}^{2}+\varepsilon^{2} \partial_{y}^{2}\right)\right) w=0 .
$$

Let $w$ be of the form $\mathcal{A}(z) \exp i\left(k_{x} x+k_{y} y-\omega t\right)$ with non-negative $k_{x}, k_{y}$ and a (complex) pulsation $\omega$. We can define a complex parameter with non-negative real part, similar to the one used by [7]:

$$
\mu^{2}=\varepsilon k^{2}-i \omega \operatorname{Re} \sqrt{\varepsilon},
$$

where $k^{2}=k_{x}^{2}+\varepsilon k_{y}^{2}$. Thanks to this notation, the solutions of (5) are such that

$$
\begin{aligned}
\mathcal{A}(z)=C_{1} \cosh \sqrt{\varepsilon} k(z-1)+C_{2} \sinh \sqrt{\varepsilon} k(z-1) & \\
& +C_{3} \cosh \mu(z-1)+C_{4} \sinh \mu(z-1) .
\end{aligned}
$$

The surface tension did not appear yet because it is only in the boundary conditions.

Up to now we have eliminated $u, v$ and $p$ only in the volumic equations. We still have to use the boundary conditions of (4) to find the conditions on the remaining field $w$.

Easy computations on the boundary conditions of (4), similar to the ones done in [12], give:

$$
\begin{aligned}
& w_{z}(0)=0 \\
& w(0)=0 \\
& w_{z z}(1)-\varepsilon w_{x x}(1)-\varepsilon^{2} w_{y y}(1)=0, \\
& w_{x x}+\varepsilon w_{y y}(1)-w_{z t t}(1)+\frac{3 \sqrt{\varepsilon}}{\operatorname{Re}}\left(w_{x x z t}+\varepsilon w_{y y z t}\right)+\frac{1}{\operatorname{Re} \sqrt{\varepsilon}} w_{z z z t}(1)=\frac{1}{\operatorname{Bo}}\left(\partial_{x}^{2}+\varepsilon \partial_{y}^{2}\right)^{2} w .
\end{aligned}
$$


The solutions (7) satisfies a homogeneous linear system in the constants $C_{1}, C_{2}, C_{3}, C_{4}$. Its matrix is:

$$
\left(\begin{array}{cccc}
\sqrt{\varepsilon} k \sinh (\sqrt{\varepsilon} k) & -\sqrt{\varepsilon} k \cosh (\sqrt{\varepsilon} k) & \mu \sinh \mu & -\mu \cosh \mu \\
\cosh (\sqrt{\varepsilon} k) & -\sinh (\sqrt{\varepsilon} k) & \cosh \mu & -\sinh \mu \\
2 k^{2} \varepsilon & 0 & \mu^{2}+\varepsilon k^{2} & 0 \\
-k^{2}-\frac{k^{4}}{\mathrm{Bo}} & \sqrt{\varepsilon} \omega^{2} k+\frac{2 i \varepsilon \omega k^{3}}{\mathrm{Re}} & -k^{2}-\frac{k^{4}}{\mathrm{Bo}} & \frac{2 \mu \sqrt{\varepsilon i \omega k^{2}}}{\mathrm{Re}}
\end{array}\right)
$$

where $k^{2}=k_{x}^{2}+\varepsilon k_{y}^{2}$. It suffices to compute its determinant to get the dispersion relation:

$$
\begin{aligned}
& 4 \varepsilon k^{2} \mu\left(\varepsilon k^{2}+\mu^{2}\right)+4 \mu k^{3} \varepsilon^{3 / 2}(\mu \sinh (k \sqrt{\varepsilon}) \sinh \mu-k \sqrt{\varepsilon} \cosh (k \sqrt{\varepsilon}) \cosh \mu) \\
&-\left(\varepsilon k^{2}+\mu^{2}\right)^{2}(\mu \cosh (k \sqrt{\varepsilon}) \cosh \mu-k \sqrt{\varepsilon} \sinh (k \sqrt{\varepsilon}) \sinh \mu) \\
&-\left(k+k^{3} / \mathrm{Bo}\right) \sqrt{\varepsilon} \operatorname{Re}^{2}(\mu \sinh (k \sqrt{\varepsilon}) \cosh \mu-k \sqrt{\varepsilon} \cosh (k \sqrt{\varepsilon}) \sinh \mu)=0 .
\end{aligned}
$$

This relation is identical to the one of [12] appart from the surface tension term and the new definition of $k$ for the 3D geometry $\left(k^{2}=k_{x}^{2}+\varepsilon k_{y}^{2}\right)$. Our process of nondimensionnalizing makes a difference between $x$ and $z$. This is why [7] have $k$ terms, and we have $k \sqrt{\varepsilon}$ terms instead.

\subsubsection{Asymptotic of the phase velocity (very large Re)}

In this subsection, we state the following Proposition concerning the phase velocity:

Proposition 1. Under the assumptions

$$
\begin{aligned}
& k \sqrt{\varepsilon} \operatorname{Re} c \rightarrow+\infty, \\
& k=O(1), \\
& \varepsilon \rightarrow 0, \\
& \operatorname{Re} \rightarrow+\infty, \\
& c=O(1) \text { (and c bounded away from } 0),
\end{aligned}
$$

Bo bounded away from 0 ,

if there exists a complex phase velocity $c=\omega / k$ solution of (10), then it is such that:

$$
c=\sqrt{\left(1+\frac{k^{2}}{\mathrm{Bo}}\right) \frac{\tanh (k \sqrt{\varepsilon})}{k \sqrt{\varepsilon}}}-\frac{e^{i \pi / 4} \operatorname{Re}^{-1 / 2}(k \sqrt{\varepsilon})^{1 / 4}}{2 \tanh ^{3 / 4}(k \sqrt{\varepsilon})}+o\left(\varepsilon^{-1 / 4} \operatorname{Re}^{-1 / 2}\right) .
$$

Moreover, the decay rate in our finite-depth geometry does not depend on the surface tension but only on the viscosity. It is:

$$
\operatorname{Im}(\omega)=\operatorname{Im}(k c)=\frac{-1}{2 \sqrt{2}} \frac{k^{5 / 4} \varepsilon^{1 / 8}}{\sqrt{\operatorname{Re}} \tanh ^{3 / 4}(k \sqrt{\varepsilon})}+o\left(\varepsilon^{-1 / 4} \operatorname{Re}^{-1 / 2}\right) .
$$

Notice that our geometry is not infinite. So our formula may be different from Boussinesq's and Lamb's.

Appart from the surface tension terms and the change of notation of $k$, the proof is not different from the one of Proposition 1 in [12].

To what extent is surface tension relevant? 


\subsection{Relevance of surface tension and viscosity}

As was explained in the previous subsection, surface tension yields only a real term in the phase velocity. So it does not give dissipation, but only dispersion. On the contrary, viscosity influences both the real and the imaginary part of the phase velocity.

We choose two different fluids (water and mercury) in order to discuss the relevance of surface tension and viscosity. Their physical parameters are listed in Table 1.

\begin{tabular}{|c|c|c|c|c|c|}
\hline & $\sigma\left(N m^{-1}\right)$ & $\rho\left(\mathrm{kg} \mathrm{m} \mathrm{m}^{-3}\right)$ & $\nu(\mathrm{Pa} s)$ & $1 / \mathrm{Re}$ & $1 / \mathrm{Bo}$ \\
\hline water $20^{\circ} \mathrm{C}$ & $7.310^{-2}$ & $10^{3}$ & $10^{-3}$ & $3.210^{-7} / h^{3 / 2}$ & $7.410^{-6} / l^{2}$ \\
mercury $20^{\circ} \mathrm{C}$ & $4.410^{-1}$ & $1.310^{4}$ & $1.5610^{-3}$ & $3.810^{-8} / h^{3 / 2}$ & $3.510^{-6} / l^{2}$ \\
\hline
\end{tabular}

Table 1: Physical parameters for two fluids

Given $\sigma, \rho, \nu$, the Re and Bo number are evaluated in Table 1 . Depending on $h$ or $l$, the Re or Bo dimensionless parameters are greater or less than 1. The critical values for each of them are given in Table 2. It appears that viscosity is not so relevant as surface tension.

\begin{tabular}{|c|c|c|}
\hline & $h_{c r i t-\mathrm{Re}}$ & $l_{\text {crit-Bo }}$ \\
\hline water $20^{\circ} \mathrm{C}$ & $4.610^{-5}$ & $2.710^{-3}$ \\
mercury $20^{\circ} \mathrm{C}$ & $1.110^{-5}$ & $1.910^{-3}$ \\
\hline
\end{tabular}

Table 2: Critical values of length (in $m$ ) for which $1 / \operatorname{Re}$ or $1 /$ Bo is 1 .

Furthermore, so as to compare 1/Bo and $\varepsilon$, and under the Boussinesq approximation, one may compute the ratio

$$
\frac{1}{\mathrm{Bo}} / \varepsilon=\frac{\sigma}{\rho g h^{2}}
$$

The critical value of $h$, for which this ratio is 1 , is $h_{a p p}=\sqrt{\sigma /(\rho g)}$. This other critical value is either $2.7 \mathrm{~mm}$ for water or $1.9 \mathrm{~mm}$ for mercury. In other words if $h>h_{\text {app }}$, then the surface tension term is irrelevant and even less important than gravity measured by $\varepsilon$ in the equation. In most current experiments, $h$ is more than $0.1 \mathrm{~m}$ and so, surface tension should (globally) not be taken into account. As D. Lannes says: "for coastal waves of characteristic length $L_{x}=10 \mathrm{~m}$, capillary effects represent only $0.0003 \%$ of the gravity effects" ([11] section 9.1.2).

Surface tension is irrelevant for flows with $h_{0}$ more than $1 \mathrm{~cm}$. Yet, even if the initial flow satisfies this, it may or may not remain true. For instance, in case of flows with ripples created by the wind or in case of wave breaking, very short wavelengths appear. See section 9.1.2 of [11] for some references.

In the case of a flow in a shallow channel (some $\mathrm{mm}$ height), and for mercury, a depression solitary wave was predicted (as early as the initial article of Korteweg and de Vries [8]) and observed in [2]. In this experiment, $A=0.064 \mathrm{~mm}, h=2.12 \mathrm{~mm}$. As a consequence, $\mathrm{Re}=2547$ and viscosity should be taken into account.

In the following, we assume 1/Bo between $O(\varepsilon)(l \sim 1 \mathrm{~mm})$ and $O\left(\varepsilon^{2}\right)(l \sim 1 \mathrm{~cm})$. 


\subsection{Viscous Boussinesq System}

Let us first discuss the regime binding $\varepsilon$, Re, Bo.

The complex phase velocity (11) contains gravitational and viscous terms that we want to compare so as to find the regime at which their variations are of the same order of magnitude. The first term is the gravitational term $\left(\sqrt{\left(1+k^{2} / \mathrm{Bo}\right) \tanh (k \sqrt{\varepsilon}) /(k \sqrt{\varepsilon})}\right)$ which may be expanded when $\varepsilon$ tends to zero: $1-k^{2} \varepsilon(1-3 /(\varepsilon \mathrm{Bo})) / 6+O\left(\varepsilon^{2}\right)+O\left(\mathrm{Bo}^{-2}\right)$. The second is purely viscous and can be expanded: $-\sqrt{2}(1+i)(4 \sqrt{k})^{-1}(\operatorname{Re} \sqrt{\varepsilon})^{-1 / 2}+$ $\left.o(\operatorname{Re} \sqrt{\varepsilon})^{-1 / 2}\right)$. So if $1 / \mathrm{Bo}=O(\varepsilon)$ or less but $1 /(\varepsilon \mathrm{Bo}) \nsim 1 / 3$, the variations of $c$ on the gravitational and on the viscous effects are of the same order of magnitude when $\varepsilon(1-3 /(\varepsilon \mathrm{Bo}))$ and $(\operatorname{Re} \sqrt{\varepsilon})^{-1 / 2}$ are of the same order. In this regime of very large Re, studied hereafter, the dependence of Re on $\varepsilon$ is such that (to simplify, we assume here $1 / \mathrm{Bo}=o(\varepsilon))$ :

$$
\operatorname{Re} \sim \varepsilon^{-5 / 2} .
$$

This would be wrong if $1 /(\varepsilon \mathrm{Bo}) \sim 1 / 3$ as is well-known for non-viscous fluids. In that case, we would need to go further in the expansion. But since it was assumed above that $1 /$ Bo is between $O\left(\varepsilon^{2}\right)$ and $O(\varepsilon)$, we are driven to choose the regime (13) and exclude $1 /(\varepsilon \mathrm{Bo}) \sim 1 / 3$.

Our main purpose here is to state an asymptotic system of reduced size from the global Navier-Stokes equations in the whole moving domain with surface tension and a predominant propagation direction.

Proposition 2. Let $\mathbf{x}=(x, y) \in \mathbb{R}^{2}$. Let $\eta(\mathbf{x}, t)$ be the free boundary's height. Let $\left(u^{b, 0}(\mathbf{x}, \gamma), v^{b, 0}(\mathbf{x}, \gamma)\right)$ for $\gamma \in(0,+\infty)\left(\operatorname{resp} .\left(u^{u, 0}(\mathbf{x}, z), v^{u, 0}(\mathbf{x}, z)\right)\right.$ for $\left.z \in(0,1+\varepsilon \eta(\mathbf{x}, t))\right)$ be the initial horizontal velocity in the boundary layer (resp. in the upper part of the domain). Let $f(\mathbf{x}, \gamma):=u^{b, 0}(\mathbf{x}, \gamma)-u^{u, 0}(\mathbf{x}, z=0)$ and $g(\mathbf{x}, \gamma):=v^{b, 0}(\mathbf{x}, \gamma)-v^{u, 0}(\mathbf{x}, z=0)$. If for any $\mathbf{x} \in \mathbb{R}^{2}, \gamma \mapsto f$ and $\gamma \mapsto g$ are uniformly continuous in $\gamma$ and $f_{x}, g_{y} \in L_{\gamma}^{1}\left(\mathbb{R}^{+}\right)$, then the solution of the Navier-Stokes equation with this given initial condition satisfies the weakly transverse Boussinesq system:

$$
\begin{aligned}
& u_{t}+\eta_{x}-\frac{\eta_{x x x}}{\mathrm{Bo}}+\varepsilon u u_{x}+\varepsilon^{3 / 2} v u_{y}-\varepsilon \eta_{x t t} \frac{\left(z^{2}-1\right)}{2}=O\left(\varepsilon^{2}\right)+O(\varepsilon / \mathrm{Bo}), \\
& v_{t}+\sqrt{\varepsilon} \eta_{y}-\frac{\sqrt{\varepsilon} \eta_{x x y}}{\mathrm{Bo}}+\varepsilon u v_{x}+\varepsilon^{3 / 2} v v_{y}-\varepsilon \eta_{y t t} \frac{\left(z^{2}-1\right)}{2}=O\left(\varepsilon^{2}\right)+O(\varepsilon / \mathrm{Bo}), \\
& \eta_{t}+u_{x}(\mathbf{x}, z, t)-\frac{\varepsilon}{2} \eta_{x x t}\left(z^{2}-\frac{1}{3}\right)+\varepsilon(u \eta)_{x}+\sqrt{\varepsilon} v_{y}+\varepsilon^{\frac{3}{2}}(v \eta)_{y}-\frac{\varepsilon}{\sqrt{\pi R}}\left(u_{x}+\sqrt{\varepsilon} v_{y}\right) * \frac{1}{\sqrt{t}} \\
& \quad+\frac{2 \varepsilon}{\sqrt{\pi}} \int_{\gamma^{\prime \prime}=0}^{+\infty} \operatorname{div}\left(\begin{array}{c}
\left(u^{b, 0}\left(\mathbf{x}, \gamma^{\prime \prime}\right)-u^{u, 0}(\mathbf{x}, z=0)\right) \\
\sqrt{\varepsilon}\left(v^{b, 0}\left(\mathbf{x}, \gamma^{\prime \prime}\right)-v^{u, 0}(\mathbf{x}, z=0)\right)
\end{array}\right) \int_{\gamma^{\prime}=0}^{\sqrt{\frac{R}{4 t}} \gamma^{\prime \prime}} e^{-\gamma^{\prime 2}} \mathrm{~d} \gamma^{\prime} \mathrm{d} \gamma^{\prime \prime}=o(\varepsilon),
\end{aligned}
$$

where the convolution, denoted with $*$, is in time, the parameters $\varepsilon$, and $R$ have been defined and $z \in(0,1+\varepsilon \eta(\mathbf{x}, t))$.

With the change of notation $k^{2}=k_{x}^{2}+k_{y}^{2}$, the whole proof is the very same as in [12] and is omitted.

Had we proceeded in the same way as [7], we would have distinguished two subdomains: the upper part $(z>\varepsilon)$ where viscosity can be neglected, and the lower part $(0<z<\varepsilon)$ which is a boundary layer at the bottom and where viscosity must be taken into account. 
The resolution in each part would have enabled to match the solution on the common boundary. Instead, we assume overlapping domains.

Remark 3. As is stressed at the non-dimensionnalizing step, there are two choices for the scale of the transverse horizontal velocity. Either one takes $V=\varepsilon^{3 / 2} c_{0}$ (see Johnson's book [5] for instance or many others), or one uses $V=\varepsilon c_{0}$ (see the article of Lannes-Saut [9] for instance). Firstly one must notice that since this field is not eliminated (as $w$ is), its scale is relevant. Moreover the latter scaling is more general than the former one because it does not assume $v$ to be $O(\sqrt{\varepsilon})$. The main difference is that $v_{\text {Lannes-Saut }}=\sqrt{\varepsilon} v_{\text {Johnson }}$, where one implicitely assumes that the fields $v_{\text {Lannes-Saut }}$ and $v_{\text {Johnson }}$ are $O(1)$. To be clear, Lannes-Saut's choice amounts to assume $v=O(1)$ although it can be written $\sqrt{\varepsilon} \partial_{y} \psi$ ! If we plug this $v=\sqrt{\varepsilon} \partial_{y} \psi$ in (14), we get the same transverse Boussinesq system as [5] (p. 210) because $\varepsilon^{3 / 2} v_{\text {Lannes-Saut }}$ transforms into $\varepsilon^{2} v_{\text {Johnson }}$ and go to $O\left(\varepsilon^{2}\right)$. So the non-dimensionnalizing process of Lannes-Saut is weaker than the one done by many other authors.

Lannes and Saut [9] give one more argument by exhibiting an (odd) solution to the linearized system (in their Remark 3) such that $v=\sqrt{\varepsilon} \partial_{y} \psi$. It is of size $O(1)$ and not $O(\sqrt{\varepsilon})$ :

$$
u=0, v=f^{\prime}(y-\sqrt{\varepsilon} t)-f^{\prime}(y-\sqrt{\varepsilon} t), \eta=f^{\prime}(y-\sqrt{\varepsilon} t)+f^{\prime}(y-\sqrt{\varepsilon} t) .
$$

We want to notice here that the potential representation of the velocity might have induced the idea that if the potential is physical, then the deduced field (the velocity) is such that $(u, v)=\left(\partial_{x} \phi, \sqrt{\varepsilon} \partial_{y} \phi\right)$ and so, that $v=O(\sqrt{\varepsilon})$. Had we started from the velocity pressure formulation, this assumption would not be natural. Yet, such an attempt of explanation does not match reality. Johnson [5] uses the velocity pression representation as we do and assumes $v=O(\sqrt{\varepsilon})$, while Lannes and Saut [9] use the potential representation and do not make this assumption!

Remark 4. It was already noticed in [12] that the double integral term in (14) is new and surprising because of its dependence on the initial condition. If we assume an initial flow of Euler type (so that $u^{b, 0}-u^{u, 0}=0$ ), the double integral vanishes. But is it physical? In other words, does an initial (inviscid) flow in the boundary layer (where Navier-Stokes applies) establish (as a Navier-Stokes flow) fast or not? Can we assume an initial Euler flow and a Navier-Stokes evolution PDE without loss of generality?

We claim that the answer is negative for at least two reasons. On the one hand, the characteristic time for the viscous effects to appear is roughly $T_{N S E}=\rho h_{0}^{2} / \nu$ or $T_{N S E}=$ $\rho l^{2} / \nu$. Then, its ratio with the characteristic time of the inviscid gravity flow $\left(l / c_{0}\right)$ is either $\operatorname{Re} \sqrt{\varepsilon}=\varepsilon^{-2}$ or $\operatorname{Re} / \sqrt{\varepsilon}=\varepsilon^{-3}$ respectively. Whatever the chosen characteristic time, this ratio is large and the flow in the boundary layer does not establish fast enough.

On the other hand, as is argued in [12], the value of this integral for a typical exponential flow in the boundary layer can be computed. It tends to zero only like $1 / \sqrt{t}$ as is classical for Navier-Stokes flows. As a consequence, one may assume that it goes from zero (Euler) to exponential (boundary layer) within the time $(1 / \sqrt{t})$ which is large with respect to the characteristic time of the flow. 


\section{Viscous KdV with surface tension}

Starting from (14), one may assume the fields do not depend on $y$, and $v=0$. So the flow is purely one dimensional (one direction). One may see that the zeroth order of this Boussinesq system is the wave equation. Then one knows that there are two waves propagating in each direction. If we look only for the waves that propagate to the right, one may make a change of variables suggested by the zeroth order equation:

$$
\xi=x-t, \tau=\varepsilon t
$$

Every term can easily be converted in these new coordinates appart from the convolution (one derivative and a half integration), the new surface tension term and the double integral. The first was treated in a very clean way in [12]. The second is new, but very simple. The third one was treated in [12], but its treatment is improved below. After a change of variable, this term writes:

$$
+\frac{2 \varepsilon}{\sqrt{\pi}} \int_{\gamma^{\prime \prime}=0}^{+\infty}\left(u_{x}^{b, 0}\left(\xi+\frac{\tau}{\varepsilon}, \gamma^{\prime \prime}\right)-u_{x}^{u, 0}\left(\xi+\frac{\tau}{\varepsilon}, z=0\right)\right) \times \int_{\gamma^{\prime}=0}^{\sqrt{\frac{R \varepsilon}{4 \tau}} \gamma^{\prime \prime}} e^{-\gamma^{\prime 2}} \mathrm{~d} \gamma^{\prime} \mathrm{d} \gamma^{\prime \prime}
$$

The article [12] argued that if the initial horizontal velocity is localized and $\tau$ not too small, then $u^{b, 0}\left(\xi+\tau / \varepsilon, \gamma^{\prime \prime}\right)-u^{u, 0}(\xi+\tau / \varepsilon, z=0)$ will be negligible in comparison with $\varepsilon$. More precisely, if $\xi \mapsto u^{b, 0}\left(\xi, \gamma^{\prime \prime}\right)-u^{u, 0}(\xi, z=0)$ tends to zero when $\xi$ tends to $+\infty$ at least as $\xi^{-1}$. Then the term $(15)$ is at least $O\left(\varepsilon^{2}\right)$.

One may add one more argument if $\tau$ is still not too small. The upper bound of the inner integral contains a $\sqrt{\varepsilon}$. So mainly large $\gamma^{\prime \prime}$ (larger than $1 / \sqrt{\varepsilon}$ ) will be relevant. But for those $\gamma^{\prime \prime}$, the $u^{b, 0}\left(\xi+\tau / \varepsilon, \gamma^{\prime \prime}\right)-u^{u, 0}(\xi+\tau / \varepsilon, z=0)$ is small uniformly in $\xi+\tau / \varepsilon$ because of the matching condition on $u^{0}$ between the boundary layer and the upper part. So (15) is small for two different reasons and we can state the viscous KdV equation with surface tension in the following Proposition.

Proposition 5. If the initial flow is localized, the KdV change of variables applied to the $1 D$ version of the system (14) leads to

$$
2 \tilde{\eta}_{\tau}+3 \tilde{\eta} \tilde{\eta}_{\xi}+\left(\frac{1}{3}-\frac{1}{\varepsilon \mathrm{Bo}}\right) \tilde{\eta}_{\xi \xi \xi}-\frac{1}{\sqrt{\pi R}} \int_{\xi^{\prime}=0}^{\tau / \varepsilon} \frac{\tilde{\eta}_{\xi}\left(\xi+\xi^{\prime}, \tau\right)}{\sqrt{\xi^{\prime}}} \mathrm{d} \xi^{\prime}=o(1),
$$

for not too small times $\tau$, where we set $R e=R \varepsilon^{-5 / 2}$.

We do not replace the upper bound of the convolution $(\tau / \varepsilon)$ by $+\infty$ for two reasons. On the one hand we do not know how fast this integral converges when $\tau / \varepsilon$ tends to $+\infty$. On the other hand the $\tau$ term reminds us that this integral on $\xi^{\prime}$ is a mixture of time and space.

\section{Viscous KP equation with surface tension}

Below, we derive the viscous KP equation, then discuss the zero-mass (in $\xi$ ) constraint. 


\subsection{Derivation}

We recall that we set $\mathrm{Re}=R \varepsilon^{-5 / 2}$, and $1 / \mathrm{Bo}=O(\varepsilon)$ but $1 /(\varepsilon \mathrm{Bo}) \neq 1 / 3+o(1)$.

With these assumptions, the change of variables

$$
\xi=x-t, y=y, \tau=\varepsilon t,
$$

and the assumption that the initial flow is localized, the system (14) writes (since $\varepsilon / \mathrm{Bo}=$ $\left.O\left(\varepsilon^{2}\right)\right)$ :

$$
\begin{gathered}
-u_{\xi}(\xi, y, z, \tau)+\eta_{\xi}+\varepsilon\left(u_{\tau}+u u_{\xi}-\eta_{\xi \xi \xi}\left(z^{2}-1\right) / 2-\frac{\eta_{\xi \xi \xi}}{\varepsilon \mathrm{Bo}}+\sqrt{\varepsilon} v u_{y}\right)=O\left(\varepsilon^{2}\right), \\
-v_{\xi}+\sqrt{\varepsilon} \eta_{y}+\varepsilon\left(v_{\tau}+u v_{\xi}-\sqrt{\varepsilon} \eta_{y \xi \xi}\left(z^{2}-1\right) / 2-\frac{\eta_{\xi \xi y}}{\sqrt{\varepsilon} \mathrm{Bo}}+\sqrt{\varepsilon} v v_{y}\right)=O\left(\varepsilon^{2}\right), \\
-\eta_{\xi}+u_{\xi}+\varepsilon\left(\eta_{\tau}+\frac{1}{2} \eta_{\xi \xi \xi}\left(z^{2}-1 / 3\right)+(u \eta)_{\xi}+\sqrt{\varepsilon}(v \eta)_{y}\right)+\sqrt{\varepsilon} v_{y} \\
-\frac{\varepsilon}{\sqrt{\pi R}} \int_{\xi^{\prime}=0}^{\tau / \varepsilon} \frac{u_{\xi}\left(\xi+\xi^{\prime}, y, z, \tau\right)+\sqrt{\varepsilon} v_{y}\left(\xi+\xi^{\prime}, y, z, \tau\right)}{\sqrt{\xi^{\prime}}} \mathrm{d} \xi^{\prime}=O\left(\varepsilon^{2}\right) .
\end{gathered}
$$

In the above system, we did not write the double integral term from (15). Indeed, it was justified in the previous section that this term can be neglected. Moreover, one might prove that the transverse velocity is such that $v(z)=v\left(z^{\prime}\right)+O(\varepsilon)$ in a similar way to Lemma 11 of [12] (roughly, one differentiate $(14)_{2}$ with respect to $z$, then integrate with respect to time $t$ ). So as to go further, since, at the first order $u_{\xi}=\eta_{\xi}$, one may justify that $u_{\tau}=\eta_{\tau}+o(1)$ as in the inviscid case, and $u=\eta+o(1)$. One may then add the first and third equation:

$$
\begin{aligned}
2 \varepsilon \eta_{\tau}+3 \varepsilon \eta \eta_{\xi}+ & \left(\frac{\varepsilon}{3}-\frac{1}{\mathrm{Bo}}\right) \eta_{\xi \xi \xi}+\varepsilon^{3 / 2} v u_{y}+\varepsilon^{3 / 2}(v \eta)_{y}+\sqrt{\varepsilon} v_{y} \\
& -\frac{\varepsilon}{\sqrt{\pi R}} \int_{\xi^{\prime}=0}^{\tau / \varepsilon} \frac{\eta_{\xi}\left(\xi+\xi^{\prime}, y, \tau\right)+\sqrt{\varepsilon} v_{y}\left(\xi+\xi^{\prime}, y, z, \tau\right)}{\sqrt{\xi^{\prime}}} \mathrm{d} \xi^{\prime}=O\left(\varepsilon^{2}\right) .
\end{aligned}
$$

In order to eliminate $v$, one must differentiate with respect to $\xi$ to use the corresponding $(17)_{2}$. Our choice of non-dimensionalization forces us to manage extra terms, but we get the classical KP equation with a viscous term:

$$
\begin{array}{r}
\left(2 \eta_{\tau}+3 \eta \eta_{\xi}+\left(\frac{1}{3}-\frac{1}{\varepsilon \mathrm{Bo}}\right) \eta_{\xi \xi \xi}\right)_{\xi}+\eta_{y y}-\frac{1}{\sqrt{\pi R}} \int_{\xi^{\prime}=0}^{\tau / \varepsilon} \frac{\eta_{\xi \xi}\left(\xi+\xi^{\prime}, y, \tau\right)}{\sqrt{\xi^{\prime}}} \mathrm{d} \xi^{\prime} \\
=-\sqrt{\varepsilon}\left(2 v \eta_{\xi y}+v_{y} \eta_{\xi}+v_{\tau y}\right)+O(\varepsilon)+O(1 / \mathrm{Bo})
\end{array}
$$

where $v$ satisfies $(17)_{2}$. This completes the proof of the following Proposition.

Proposition 6. If the initial flow is localized, in the Boussinesq approximation and if $1 / \mathrm{Bo}=O(\varepsilon)$ but $1 / \mathrm{Bo} \neq \varepsilon / 3+o(\varepsilon)$, the surface waves of a viscous fluid propagating predominantly along $x$ and weakly along $y$ satisfy the viscous KP equation:

$$
\left(2 \eta_{\tau}+3 \eta \eta_{\xi}+\left(\frac{1}{3}-\frac{1}{\varepsilon \mathrm{Bo}}\right) \eta_{\xi \xi \xi}\right)_{\xi}+\eta_{y y}-\frac{1}{\sqrt{\pi R}} \int_{\xi^{\prime}=0}^{\tau / \varepsilon} \frac{\eta_{\xi \xi}\left(\xi+\xi^{\prime}, y, \tau\right)}{\sqrt{\xi^{\prime}}} \mathrm{d} \xi^{\prime}=O(\sqrt{\varepsilon})
$$


or the equivalent mixed system

$$
\begin{aligned}
2 \eta_{\tau}+3 \eta \eta_{\xi}+\left(\frac{1}{3}-\frac{1}{\varepsilon \mathrm{Bo}}\right) \eta_{\xi \xi \xi}-\frac{1}{\sqrt{\pi R}} \int_{\xi^{\prime}=0}^{\tau / \varepsilon} \frac{\eta_{\xi}\left(\xi+\xi^{\prime}, y, \tau\right)}{\sqrt{\xi^{\prime}}} \mathrm{d} \xi^{\prime}+v_{y}=O(\sqrt{\varepsilon}), \\
-v_{\xi}+\eta_{y}=O(\sqrt{\varepsilon}) .
\end{aligned}
$$

One must keep in mind that our choice of scale triggers the $\sqrt{\varepsilon}$ (even inside the convolution) in (17). This choice is already discussed in Remark 3. Had we made the same choice of scale for $v$ as Johnson [5] and many others, we would have replaced $v$ by $\sqrt{\varepsilon} v_{J}$ in (17). The equivalent (18) could be simplified by $\varepsilon$ and it would write :

$$
2 \eta_{\tau}+3 \eta \eta_{\xi}+\left(\frac{1}{3}-\frac{1}{\varepsilon \mathrm{Bo}}\right) \eta_{\xi \xi \xi}+v_{J y}-\frac{1}{\sqrt{\pi R}} \int_{\xi^{\prime}=0}^{\tau / \varepsilon} \frac{\eta_{\xi}\left(\xi+\xi^{\prime}, y, \tau\right)}{\sqrt{\xi^{\prime}}} \mathrm{d} \xi^{\prime}=O(\varepsilon),
$$

and $v_{J}$ would satisfy $(17)_{2}$ modified:

$$
-v_{J \xi}+\eta_{y}+\varepsilon\left(v_{J \tau}+u v_{J \xi}-\eta_{y \xi \xi}\left(z^{2}-1\right) / 2-\frac{\eta_{\xi \xi y}}{\varepsilon \mathrm{Bo}}\right)=O\left(\varepsilon^{3 / 2}\right)
$$

Oddly, this would lead to the same KP equation as (19) but up to the order $O(\varepsilon)$ and not $O(\sqrt{\varepsilon})$. During the proof, we exhibit the $O(\sqrt{\varepsilon})$ terms which would be $O(\varepsilon)$ if $v=\sqrt{\varepsilon} v_{J}$ although they are not kept in the Proposition. So there is no contradiction and the accuracy of $\mathrm{KP}$ is tied to the property that $v=O(\sqrt{\varepsilon})$ or not.

\subsection{The zero-mass constraint}

As is very well discussed in [17], the usual KP equation is often written with an operator $\partial_{\xi}^{-1} \partial_{y}^{2}$. Yet such an operator assumes the solution is differentiated (in $\xi$ ) from a function that tends to 0 when $\xi \rightarrow \pm \infty$. It is proved in [17] that although it is not obvious, this assumption is right for inviscid fluids. Their proof is done in two steps.

In the first step, the linear KP equation is solved with inverse Fourier transforms. For more general equations, but only dispersive, like $u_{t}-L u_{\xi}+\partial_{\xi}^{-1} \partial_{y y} u=0$ (the symbol of $L$ is $\varepsilon|X|^{\alpha}$ for $\left.\alpha>1 / 2\right)$, the fundamental solution writes

$$
G(t, \xi, y)=\mathcal{F}_{\left(X, y^{\prime}\right) \rightarrow(\xi, y)}^{-1}\left[e^{i t\left(\varepsilon X|X|^{\alpha}-y^{\prime 2} / X\right)}\right]
$$

where $\varepsilon= \pm 1$ depends on the KPI or KPII equation (or on the sign of $1 / 3-1 /(\varepsilon \mathrm{Bo})$ ). For the usual KPI and KPII, $\alpha=2$.

Thanks to the Lebesgue's Dominated convergence theorem and inventive changes of variables, it is proved that the fundamental solution is regular, and is differentiated from a more regular function $A$ (which is $\mathcal{C}\left(\mathbb{R}^{2}\right) \bigcap L^{\infty}\left(\mathbb{R}^{2}\right) \bigcap \mathcal{C}_{\xi}^{1}\left(\mathbb{R}^{2}\right)$ ) such that $A * u_{0} \rightarrow 0$ when $|\xi| \rightarrow+\infty$.

In a second step, the nonlinearity is treated with the Duhamel formulation. The solution is then the convolution of the group of the linear equation with the nonlinear term $u u_{\xi}$ and all what is already proved for the linear equation extends to the nonlinear one. 
One may wonder whether such an interesting result can extend to the viscous case. Indeed, the proof is even simpler and the details are left to the interested reader. The Fourier transform of the dissipative term writes

$$
\begin{aligned}
\frac{-1}{\sqrt{\pi R}} \mathcal{F}_{\xi \rightarrow X}\left(\int_{0}^{+\infty} \frac{\eta_{\xi}\left(\xi+\xi^{\prime}, y, \tau\right)}{\sqrt{\xi^{\prime}}} \mathrm{d} \xi^{\prime}\right) & =\frac{-1}{\sqrt{2 \pi^{2} R}} \int_{\mathbb{R}_{\xi}} \int_{\xi^{\prime}=0}^{+\infty} \frac{\eta_{\xi}\left(\xi+\xi^{\prime}\right)}{\sqrt{\xi^{\prime}}} e^{-i \xi X} \mathrm{~d} \xi^{\prime} \mathrm{d} \xi \\
& =-\frac{i X}{\sqrt{\pi R}} \hat{\eta}(X) \int_{\xi^{\prime}=0}^{+\infty} \frac{e^{i \xi^{\prime} X}}{\sqrt{\left|\xi^{\prime}\right|}} \mathrm{d} \xi^{\prime} .
\end{aligned}
$$

By a simple change of variable, one may see that the last integral is indeed only a function of the sign of $X($ denoted $\operatorname{sgn}(X))$ times $1 / \sqrt{|X|}$. This function of $\operatorname{sgn}(X)$ can be computed :

$$
\int_{\xi=0}^{+\infty} \frac{e^{i \operatorname{sgn}(X) \xi}}{\sqrt{|\xi|}} \mathrm{d} \xi=\sqrt{\frac{\pi}{2}}(1+i \operatorname{sgn}(X)),
$$

and the Fourier corresponding term reads:

$$
-i \hat{\eta}(X) \frac{X}{\sqrt{2 R|X|}}(1+i \operatorname{sgn}(X))
$$

As a consequence, one could study the fundamental solution

$$
G^{N S}(t, \xi, y)=\mathcal{F}_{\left(X, y^{\prime}\right) \rightarrow(\xi, y)}^{-1}\left[e^{i t\left(\varepsilon X|X|^{\alpha}-y^{\prime 2} / X+(1+i \operatorname{sgn}(X)) X(2 R|X|)^{-1 / 2}\right)}\right] .
$$

The only real part (non-dispersive) inside the exponential is it $\times i \operatorname{sgn}(X) X(2 R|X|$ )$^{-1 / 2}=-t|X|^{1 / 2}(2 R)^{-1 / 2}$ which sign is compatible with the dissipation and ensures convergence of all the integrals. So one do not even need to use the Lebesgue dominated convergence theorem and the proof is simpler than in [17].

\section{Viscous Boussinesq equation with surface tension}

The Boussinesq equation is a second order equation that takes into account waves going both to the right and to the left. Since the derivation is straightforward, we only sketch it, following Johnson [5] (p. 216-219) and state the Proposition 7. Johnson [5] uses a different scaling, but only along the transverse direction which is not used here.

Starting from the Boussinesq system (14) written only in 1D (with $x, z, t$ and not $x, y, z, t)$, one may differentiate $(14)_{3}$ with respect to $t$ and $(14)_{1}$ with respect to $x$. The difference of these two equations writes after some easy computations:

$$
\begin{aligned}
\eta_{t t}-\eta_{x x}-\varepsilon & \left(u^{2}+\frac{\eta^{2}}{2}\right)_{x x}-\frac{\varepsilon}{3} \eta_{x x t t}+\frac{\eta_{x x x x}}{\mathrm{Bo}}+\frac{\varepsilon}{\sqrt{\pi R}} \eta_{t t} * \frac{1}{\sqrt{t}} \\
& +\frac{\varepsilon}{\sqrt{\pi R}} \text { p.v. } \frac{1}{\sqrt{t}}\left[u_{x}^{u, 0}(x, z=0)-\int_{\gamma^{\prime}=0}^{+\infty} u_{x \gamma}^{b, 0}\left(x, \gamma^{\prime}\right) e^{-\frac{R}{4 t} \gamma^{\prime 2}} \mathrm{~d} \gamma^{\prime}\right]=O\left(\varepsilon^{2}\right),
\end{aligned}
$$


where the convolution is in time and p.v. denotes the principal value as defined in the theory of distributions. The p.v. stems from an integration by parts in which one has a boundary integral

$$
\frac{\varepsilon}{\sqrt{\pi R t}}\left[f_{x}^{0}\left(x, \gamma^{\prime}\right) e^{-\frac{R \gamma^{\prime 2}}{4 t}}\right]_{\gamma^{\prime}=0}^{+\infty} .
$$

The $u^{u, 0}$ and $u^{b, 0}$ are the initial values of $u^{u}$ and $u^{b}$. They must be provided. Appart from the two viscous terms and the term generated by surface tension, this equation is identical to (3.41) of [5]. Since we concentrate on $x=O(1)$, we may write $u=\int_{-\infty}^{x} u_{x}=-\int_{-\infty}^{x} \eta_{t}\left(x^{\prime}, t\right) \mathrm{d} x^{\prime}+O(\varepsilon)$. The above equation is then the Eulerian form of the Boussinesq equation rewritten in (23).

Let us now come back to the Lagrangian coordinates and change of fields. In [5], the author proposes $X=x+\varepsilon \int_{-\infty}^{x} \eta\left(x^{\prime}, t\right) \mathrm{d} x^{\prime}$, but we consider the following change of variable easier to justify:

$$
\begin{aligned}
X & =x-\varepsilon \int_{0}^{t} u\left(x, t^{\prime}\right) \mathrm{d} t^{\prime} \\
H(X, t) & =H\left(x-\varepsilon \int_{0}^{t} u\left(x, t^{\prime}\right) \mathrm{d} t^{\prime}, t\right)=\eta(x, t)-\varepsilon \eta^{2}(x, t) .
\end{aligned}
$$

The function $H$ is assumed to be defined on $X$ in the initial domain. Using these definitions, it is straightforward to rewrite (21) under the form of a Lagrangian Boussinesq equation with surface tension and viscosity taken into account. Only the term containing the initial horizontal velocities needs some insight. It is the last one in (21) and both $u^{u, 0}(x, z=0)$ and $u_{x \gamma}^{b, 0}\left(x, \gamma^{\prime}\right)$ can be considered as depending on $x$ or on $X$ since an $\varepsilon$ appears in front of the whole term and the difference between $x$ and $X$ is $O(\varepsilon)$. The following Proposition states the two forms of the Boussinesq equation in 1D.

Proposition 7. Let a flow of a viscous fluid with surface tension in a $2 D$ channel with a flat bottom like the one depicted in Figure 1. Let $u^{u, 0}(x, z=0)$ denote the initial horizontal velocity in the upper part (respectively $u^{b, 0}(x, \gamma)$ in the boundary layer). We assume the Boussinesq approximation and $1 / \mathrm{Bo}=O(\varepsilon)$. Under these assumptions, the surface waves obey the viscous Boussinesq equation in the Eulerian form:

$$
\begin{aligned}
\eta_{t t}-\eta_{x x}-\varepsilon\left(u^{2}\right. & \left.+\eta^{2} / 2\right)_{x x}-\left(\frac{\varepsilon}{3}-\frac{1}{\mathrm{Bo}}\right) \eta_{x x x x}+\frac{\varepsilon}{\sqrt{\pi R}} \eta_{t t} * \frac{1}{\sqrt{t}} \\
& +\frac{\varepsilon}{\sqrt{\pi R}} \mathrm{p} \cdot \mathrm{v} \cdot \frac{1}{\sqrt{t}}\left(-\left(\eta_{t}\right)_{t=0}-\int_{\gamma^{\prime}=0}^{+\infty} u_{x \gamma}^{b, 0}\left(x, \gamma^{\prime}\right) e^{-\frac{R \gamma^{\prime 2}}{4 t}} \mathrm{~d} \gamma^{\prime}\right)=O\left(\varepsilon^{2}\right),
\end{aligned}
$$

where the convolution is in time and p.v. denotes the principal value as defined in the theory of distributions. The Lagrangian form (with (22)) writes:

$$
\begin{aligned}
H_{t t}(X, t)- & H_{X X}-\frac{3}{2} \varepsilon\left(H^{2}\right)_{X X}-\left(\frac{\varepsilon}{3}-\frac{1}{\mathrm{Bo}}\right) H_{X X X X}+\frac{\varepsilon}{\sqrt{\pi R}} H_{t t} * \frac{1}{\sqrt{t}} \\
& +\frac{\varepsilon}{\sqrt{\pi R}} \text { p.v. } \frac{1}{\sqrt{t}}\left[-\left(H_{t}(X, t)\right)_{t=0}-\int_{\gamma^{\prime}=0}^{+\infty} u_{x \gamma}^{b, 0}\left(x, \gamma^{\prime}\right) e^{-\frac{R}{4 t} \gamma^{\prime 2}} \mathrm{~d} \gamma^{\prime}\right]=O\left(\varepsilon^{2}\right),
\end{aligned}
$$

where the initial velocities defined on $x$ can be evaluated either on $x$ or on $X$.

Notice that the formulae $(23,24)$ also have a formulation in terms of $u^{u, 0}(x, z=0)-$ $u^{b, 0}(x, \gamma)$ which is physically meaningful. 


\section{Is the Boussinesq system consistent with Navier-Stokes ?}

The weakly transverse Boussinesq system is stated in Proposition 2. We intend to justify here that it is consistent with the initial Navier-Stokes equations (3).

What is consistency? In [12], the author justifies, but does not prove in any functionnal space, that if there is a solution to Navier-Stokes equations, with a free boundary and a flat bottom, then, under the Bousinesq approximation, the solution satisfies the viscous isotropic Boussinesq system. Such a necessary result deserves to be completed by a sufficient one which is consistency. This is the goal of the present section.

Notice that the consistency as defined by Lannes in [11] (Definition 5.1) is much more rigorous. While we use only expansions, Lannes asked whether a solution to the asymptotic model (assuming it exists), in the asymptotic regime, satisfied the initial complete model on a convenient time-interval and in convenient norms, up to a given power of the small parameter. Lannes proved in [10] that KP solution (with his scaling) is consistent with the Boussinesq system.

We prove below the following Proposition in the 1D case.

Proposition 8. Let $u^{u}(\mathbf{x}, z, t)$ be the horizontal velocity in the upper part of a flow in a geometry as in Figure 1 , and $\eta(\mathbf{x}, t)$ be the height of the free boundary above the fluid, for $\mathbf{x}=(x, y) \in \mathbb{R}^{2}, z \in(\varepsilon, 1+\varepsilon \eta(\mathbf{x}, t))$ and nonnegative time $t$. Let $\left(u^{u, 0}(\mathbf{x}, z), v^{b, 0}(\mathbf{x}, z)\right)$ denote the initial horizontal velocity in the upper part (respectively $\left(u^{b, 0}(\mathbf{x}, \gamma), v^{b, 0}(\mathbf{x}, \gamma)\right)$ in the boundary layer with $\gamma=z / \varepsilon)$.

If $\left(u^{u}, v^{u}, \eta\right)$ satisfies the non-isotropic Boussinesq system (14), then there exist fields in the upper part $\left(u^{u}, v^{u}, w^{u}, \eta\right)$ (resp. $\left(u^{b}, v^{b}, w^{b}, \eta\right)$ ) satisfying the Navier-Stokes equations (3) in the upper part (resp. the boundary layer) of the domain and the interface continuity at least as $O(\varepsilon)$.

Proof. We start from the final Boussinesq model (14) written in 1D:

$$
\begin{aligned}
u_{t}^{u}+\eta_{x}-\frac{\eta_{x x x}}{\mathrm{Bo}}+\varepsilon u^{u} u_{x}^{u}-\varepsilon \eta_{x t t} \frac{\left(z^{2}-1\right)}{2} & =O\left(\varepsilon^{2}\right)+O(\varepsilon / \mathrm{Bo}), \\
\eta_{t}+u_{x}^{u}(x, z, t)-\frac{\varepsilon}{2} \eta_{x x t}\left(z^{2}-\frac{1}{3}\right)+\varepsilon\left(u^{u} \eta\right)_{x}-\frac{\varepsilon}{\sqrt{\pi R}} u_{x}^{u} * \frac{1}{\sqrt{t}} & \\
+\frac{2 \varepsilon}{\sqrt{\pi}} \int_{\gamma^{\prime \prime}=0}^{+\infty}\left(u_{x}^{b, 0}\left(x, \gamma^{\prime \prime}\right)-u_{x}^{u, 0}(x, z=0)\right) \int_{\gamma^{\prime}=0}^{\sqrt{\frac{R}{4 t}} \gamma^{\prime \prime}} e^{-\gamma^{\prime 2}} \mathrm{~d} \gamma^{\prime} \mathrm{d} \gamma^{\prime \prime} & =o(\varepsilon),
\end{aligned}
$$

where $u^{u}$ is the horizontal velocity in the upper part $(z \in(\varepsilon, 1+\varepsilon \eta(x, t))), \eta(x, t)$ is the free boundary's height, $u^{u, 0}(x, z)$ (resp. $\left.u^{b, 0}(x, \gamma)\right)$ is the initial horizontal velocity in the upper part (resp. in the boundary layer). In the boundary layer, we set $z=\varepsilon \gamma$.

First, one must justify that $\eta_{t t}=\eta_{x x}+O(\varepsilon)$, which is easy from the zeroth order of $(25)_{1}$. Then, the equation $(25)_{1}$ enables to prove the following Lemma.

Lemma 9. A localized solution of (25) 1 is such that

$$
\begin{aligned}
\int_{0}^{1} u & =u(x, z, t)-\varepsilon \eta_{x t}\left(z^{2}-1 / 3\right) / 2+O\left(\varepsilon^{2}\right), \\
u(x, 0, t) & =u(x, z, t)-\varepsilon \eta_{x t} z^{2} / 2+O\left(\varepsilon^{2}\right), \\
u(x, 1, t) & =u(x, z, t)+\varepsilon \eta_{x t}\left(1-z^{2}\right) / 2+O\left(\varepsilon^{2}\right) .
\end{aligned}
$$


The way to prove this Lemma is identical to the proof of Lemma 11 of [12]. One differentiates $(25)_{1}$ with respect to $z$, integrate with respect to time $t$, prove that the constants of integration vanish if the wave is localized, and use that $\eta_{t t}=\eta_{x x}+O(\varepsilon)$ to get $u_{z}=\varepsilon \eta_{x t} z+O\left(\varepsilon^{2}\right)$. Completing the proof is then easy.

Thanks to the previous Lemma, if we define

$$
\begin{aligned}
p^{u}(x, z, t) & =\left(\eta-\eta_{x x} / \mathrm{Bo}\right)-\varepsilon \eta_{t t}(z-1)+\varepsilon \int_{1}^{z} \int_{1}^{z^{\prime}} u_{x t}^{u}\left(x, z^{\prime \prime}, t\right) \mathrm{d} z^{\prime \prime} \mathrm{d} z^{\prime} \\
w^{u}(x, z, t) & =\varepsilon\left(\eta_{t}+\varepsilon \eta_{x} u^{u}(z=1+\varepsilon \eta)\right)-\varepsilon \int_{1+\varepsilon \eta}^{z} u_{x}^{u},
\end{aligned}
$$

then $\left(u^{u}, w^{u}, p^{u}\right)$ satisfies $(3)_{1,3,5,6}$ up to $O\left(\varepsilon^{2}\right)+O(\varepsilon / \mathrm{Bo})$, and $(3)_{4}$ exactly in the upper part.

We must now check (3) in the boundary layer, where we define $z=\varepsilon \gamma$ and

$$
\begin{aligned}
u^{b}(x, \gamma, t)= & u^{u}(x, z=0, t)+\frac{\sqrt{R}}{2} \int_{0}^{+\infty} f_{0}\left(x, \gamma^{\prime}\right) \frac{e^{-\frac{R\left(\gamma^{\prime}-\gamma\right)^{2}}{4 t}}}{\sqrt{\pi t}} \mathrm{~d} \gamma^{\prime} \\
& -u^{u}(x, 0, .) * \mathcal{L}_{p \rightarrow t}^{-1}\left(e^{-\sqrt{R p} \gamma}\right)-\frac{\sqrt{R}}{2} \int_{0}^{+\infty} f_{0}\left(x, \gamma^{\prime}\right) \frac{e^{\frac{-R\left(\gamma^{\prime}+\gamma\right)^{2}}{4 t}}}{\sqrt{\pi t}} \mathrm{~d} \gamma^{\prime},
\end{aligned}
$$

where $f_{0}(x, \gamma)=u^{b, 0}(x, \gamma)-u^{u, 0}(x, z=0)$. It is proved in Lemma 6 of [12] that this function satisfies

$$
\begin{aligned}
\left(u^{b}-u^{u}(z=0)\right)_{t}-\left(u^{b}-u^{u}(z=0)\right)_{\gamma \gamma} / R & =0, \\
u_{t}^{b}+\eta_{x}-u_{\gamma \gamma}^{b} / R-\eta_{x x x} / \mathrm{Bo} & =O(\varepsilon)+O(\varepsilon / \mathrm{Bo}) .
\end{aligned}
$$

This $u^{b}$ also satisfies the initial conditions and the boundary conditions:

$$
\left\{\begin{aligned}
u^{b}(x, \gamma, t=0) & =u^{b, 0}(x, \gamma), \\
u^{b}(x, \gamma=0, t) & =0 \\
u^{b}(x, \gamma \rightarrow+\infty, t) & =u^{u}(x, z=0, t) \text { (continuity condition). }
\end{aligned}\right.
$$

The function $u^{b}$ is defined as $u^{b}-u^{u}(z=0)$ being the solution to the heat equation. Because it is only the zeroth order of the conservation of momentum in the boundary layer $(3)_{1}$, and also because the limit between the boundary layer and the upper part is not made sufficiently precise through the lift "function" $u^{u}(x, z=0, t)$, one may not prove more.

If we define:

$$
\begin{aligned}
p^{b}(x, \gamma, t) & =\eta(x, t)-\eta_{x x} / \mathrm{Bo}+\varepsilon \eta_{x x} / 2 \\
w^{b}(x, \gamma, t) & =-\varepsilon^{2} \int_{0}^{\gamma} u_{x}^{b}\left(x, \gamma^{\prime}, t\right) \mathrm{d} \gamma^{\prime},
\end{aligned}
$$

then $(3)_{1}$ in the boundary layer reduces to:

$$
\begin{aligned}
& \left(u^{b}-u^{u}\right)_{t}-\left(u^{b}-u^{u}\right)_{\gamma \gamma} / R-\varepsilon u^{u} u_{x}^{u}+\varepsilon u^{b} u_{x}^{b}-\varepsilon u_{\gamma}^{b} \int_{0}^{\gamma} u_{x}^{b}+\varepsilon \eta_{x x x} / 2+O\left(\varepsilon^{2}\right)+O(\varepsilon / \mathrm{Bo}), \\
& =-\varepsilon u^{u} u_{x}^{u}+\varepsilon u^{b} u_{x}^{b}-\varepsilon u_{\gamma}^{b} \int_{0}^{\gamma} u_{x}^{b}+\varepsilon \eta_{x x x} / 2+O\left(\varepsilon^{2}\right)+O(\varepsilon / \mathrm{Bo})=O(\varepsilon),
\end{aligned}
$$

where $u^{u}$ is evaluated at $z=0$. So $\left(u^{b}, w^{b}, p^{b}\right)$ satisfies $(3)_{1}$ only up to $O(\varepsilon)$. So as to go further, one should use correctors, solve a nonlinear heat equation, make a more precise study at the interface between the two subdomains and use functionnal spaces. Simple computations prove that $\left(u^{b}, w^{b}, p^{b}\right)$ satisfies $(3)_{3,4,7}$ up to $O\left(\varepsilon^{2}\right)+O(\varepsilon / \mathrm{Bo})$.

The overlapping of the boundary layer and the upper part makes that consistency cannot be justified more precisely than $O(\varepsilon)$. Yet, since the interface condition (for instance at $z=\sqrt{\varepsilon}$ ) between these two domains is satisfied, the proof is complete, up to order $O(\varepsilon)$. 


\section{Conclusion}

We modeled the water waves flow by the Navier-Stokes equations in a 3D geometry with a flat bottom and free surface. We assumed the order of magnitude of the velocity in the transverse direction and deduced the scaling in this coordinate and discussed its influence on the results (Remark 3). The linear theory gave a new dispersion relation and a new phase velocity (Proposition 1). Surface tension was discussed and seemed often more relevant than viscosity, although these two parameters act very differently. We stated the associated Boussinesq system. This intermediate system enabled us to derive the KdV equation (Proposition 5) and the KP equation or system (Proposition 6). We justified why the zero-mass constraint does not raise any trouble for viscous KP, using [17]. Using the previous computations, we derived the Boussinesq equation (Proposition 7). Reciprocally, we proved that, should they exist, the solutions to the non-isotropic Boussinesq system are consistent with the Navier-Stokes equations (Proposition 8). These systems and equations are derived for a viscous fluid and take the surface tension into account.

\section{References}

[1] J. Boussinesq, Lois de l'extinction d'une houle simple en haute mer, C. R. Math. Acad. Sci. Paris 121 (1895) 1, pp. 15-19.

[2] E. Falcon and C. Laroche and S. Fauve, Observation of depression solitary waves on a thin fluid layer, Phys. Rev. Lett. 89 (2002), 204501.

[3] H. Hasimoto and H. Ono, Nonlinear Modulation of Gravity Waves, Journal of the Physical Society of Japan, Volume 33, Issue 3, pp. 805-811 (1972).

[4] T. Iguchi, A mathematical justification of the forced Korteweg-de Vries equation for capillary-gravity waves, Kyushu J. Math., 60 (2006), 267-303.

[5] R.S. Johnson, A modern introduction to the mathematical theory of water waves Cambridge University Press, Cambridge, 1997.

[6] B.B. Kadomtsev and V.I. Petviashvili, On the stability of solitary waves in weakly dispersive media, Sov. Phys. Dokl. 15: 539-541 (1970).

[7] T. Kakutani and K. Matsuuchi, Effect of viscosity of long gravity waves, J. Phys. Soc. Japan 39 (1975) 1 pp. 237-246.

[8] D. J. Korteweg and G. de Vries, On the change of form of long waves advancing in a rectangular canal, and on a new type of long stationnary waves, London, Edinburgh Dublin Philos. Mag. J. Sci. vol. 39, 422-443 (1895).

[9] D. Lannes and J.C. Saut, Weakly transverse Boussinesq systems and the KadomtsevPetviashvili approximation. Nonlinearity 19 (2006), no. 12, 2853-2875. 
[10] D. Lannes, Consistency of the KP Approximation, Proceedings of the 4th International Conference on Dynamical Systems and Differential Equations, May 24-27, 2002, Wilmington, NC, USA, Discrete Contin. Dyn. Syst. 2003, suppl., 517-525.

[11] D. Lannes, The Water Waves Problem: Mathematical Analysis and Asymptotics, AMS, Vol. 188, 2013.

[12] H.V.J. Le Meur, Derivation of a viscous Boussinesq system for surface water waves, Asymptotic Analysis 94 (2015) pp. 309-345.

[13] P.L.-F. Liu and A. Orfila, Viscous effects on transient long-wave propagation, J. Fluid Mech. 520 (2004) pp. 83-92.

[14] C.C. Mei and P.L.-F. Liu, The damping of surface gravity waves in a bounded liquid. Journal of Fluid Mechanics 59 (1), (1973) 239 - 256.

[15] M. Ming and P. Zhang and Z. Zhang, Large time well-posedness of the threedimensional capillary-gravity waves in the long wave regime. Arch. Ration. Mech. Anal. 204 (2012), no. 2, 387-444.

[16] M. Ming and P. Zhang and Z. Zhang, Long-wave approximation to the 3-D capillarygravity waves. SIAM J. Math. Anal. 44 (2012), no. 4, 2920-2948.

[17] L. Molinet and J.C. Saut and N. Tzvetkov, Remarks on the mass constraint for KP-type equations. SIAM J. Math. Anal. 39 (2007), no. 2 pp. 627-641

[18] G.G. Stokes, Supplement to a paper on the theory of oscillatory waves, Mathematical and Physical Papers, Volume I, Cambridge University Press, (1880) pp. 314-326 